\title{
Ulcerated and Verrucous Plaque on the Chest
}

\author{
Mahdieh Fazel Jedlowski, MD, PharmD; Patrick Michael Jedlowski, MD
}

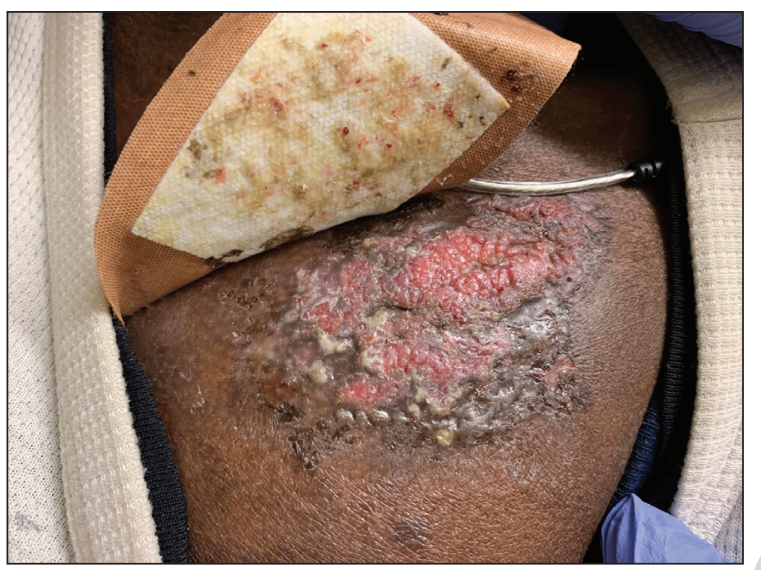

A 36-year-old man presented to an emergency department in the southwestern United States with a cough, fatigue, and worsening back pain associated with night sweats of 1 month's duration. He experienced a 9.07-kg weight loss, as well as development of a rough, nontender, nonpruritic rash along the left upper chest over the prior month. The patient was born in West Africa and reported that he had moved to the southwestern United States from the eastern United States approximately 6 years prior to presentation. Physical examination on admission revealed a $5 \times 3-c m$, purple-brown, verrucous plaque with a central pink cobblestone appearance and ulceration. Chest radiography was notable for perihilar adenopathy with no focal infiltrates or cavitary lesions. Computed tomography and magnetic resonance imaging of the chest were notable for miliary nodules throughout the lungs; extensive lytic spine lesions of cervical, thoracic, and lumbar vertebral bodies and left twelfth rib; and a left paraspinal thoracic epidural soft tissue phlegmon. Initial laboratory investigations revealed peripheral eosinophilia without absolute leukocytosis and a microcytic anemia.

\section{WHAT'S YOUR DIAGNOSIS?}
a. cutaneous leishmaniasis
b. disseminated blastomycosis
c. disseminated coccidioidomycosis
d. disseminated histoplasmosis
e. lupus vulgaris

PLEASE TURN TO PAGE E11 FOR THE DIAGNOSIS

From the University of Arizona College of Medicine, Tucson.

The authors report no conflict of interest.

Correspondence: Patrick Michael Jedlowski, MD, 7165 N Pima Canyon Dr, Tucson, AZ 85718 (pjedlowski@email.arizona.edu). 


\section{THE DIAGNOSIS:}

\section{Disseminated Coccidioidomycosis}

$\Lambda$ 6-mm punch biopsy was performed at the periphery of the ulcerated cutaneous lesion on the chest revealing extensive spherules. Serum antibody immunodiffusion for histoplasmosis and blastomycoses both were negative; however, B-D-glucan assay was positive at $364 \mathrm{pg} / \mathrm{mL}$ (reference range: $<60 \mathrm{pg} / \mathrm{mL}$, negative). Initial HIV-1 and HIV-2 antibody and antigen testing was negative as well as repeat testing at 3 weeks. Immunodiffusion for Coccidioides IgM and IgG was positive, and cocci antibody IgG complement fixation assays were positive at titers of 1:64 (reference range: $<1: 2$, negative). A computed tomography needle-guided biopsy of the paravertebral soft tissue was performed. Gram stains and bacterial cultures of the biopsies were negative; however, fungal cultures were notable for growth of Coccidioides. Given the pertinent testing, a diagnosis of disseminated coccidioidomycosis was made.

Cutaneous coccidioidomycosis can occur in 3 situations: direct inoculation (primary cutaneous coccidioidomycosis), disseminated infection (disseminated cutaneous coccidioidomycosis), or as a reactive component of pulmonary infection..$^{1,2}$ Of them, primary and disseminated cutaneous coccidioidomycosis are organism specific and display characteristic spherules and fungus on histopathology and cultures, respectively. Reactive coccidioidomycosis differs from organism-specific disease, as it does not contain spherules in histopathologic sections of tissue biopsies. ${ }^{1}$ Reactive skin manifestations occur in $12 \%$ to $50 \%$ of patients with primary pulmonary infection and include erythema nodosum, erythema multiforme, acute generalized exanthema, reactive interstitial granulomatous dermatitis, and Sweet syndrome. ${ }^{3}$

Organism-specific cutaneous coccidioidomycosis most often is correlated with hematogenous dissemination of primary pulmonary disease rather than direct inoculation of skin. ${ }^{1}$ The skin is the most common site of extrapulmonary involvement in disseminated coccidioidomycosis, and cutaneous lesions have been reported in $15 \%$ to $67 \%$ of cases of disseminated disease. ${ }^{1,4}$ In cutaneous disseminated disease, nodules, papules, macules, and verrucous plaques have been described. In a case series of disseminated cutaneous coccidioidomycosis, nodules were the most common cutaneous presentation and occurred in $39 \%$ (7/18) of patients, while verrucous plaques were the rarest and occurred in only $6 \%(1 / 18)$ of patients. ${ }^{5}$

The rate of coccidioidomycosis dissemination varies based on exposure and patient characteristics. Increased rates of dissemination have been reported in patients of African and Filipino descent, along with individuals that are immunosuppressed due to disease or medical therapy. Dissemination is clinically significant, as patients with multifocal dissemination have a greater than $50 \%$ risk for mortality. ${ }^{6}$

Disseminated coccidioidomycosis is a relatively rare manifestation of Coccidioides infection; approximately $1.6 \%$ of patients exposed to and infected with Coccidioides ultimately will develop systemic or disseminated disease. ${ }^{7,8}$ Although the rates of primary pulmonary infection are similar between patients of varying ethnicities, the rates of dissemination are higher in patients of African and Filipino ethnicity. ${ }^{8}$ In population studies of coccidioidomycosis $(\mathrm{N}=332)$, Black patients represented $33.3 \%(4 / 12)$ of disseminated cases but only $8.7 \%$ of Coccidioides cases overall. ${ }^{7}$

Population studies of Black patients with coccidioidomycosis have shown a 4 -fold higher predisposition for severe disease compared to mild disease. ${ }^{9}$ Spondylitis and meningitis also are disproportionately more common in Black patients. ${ }^{8}$ Black patients comprised $75 \%$ of all spondylitis cases in a cohort where only $25 \%$ of patients were Black. Additionally, 33\% of all meningitis cases occurred in Black patients in a cohort where $8 \%$ of total cases were Black patients. ${ }^{8}$ Within the United States, the highest rates of coccidioidomycosis meningitis are seen in Black patients. ${ }^{10}$

The pathophysiology underlying the increased susceptibility of individuals of African or Filipino descent to disseminated and severe coccidioidomycosis remains unknown. ${ }^{8}$ The level of vulnerability within this patient population has no association with increased environmental exposure or poor immunologic response to Coccidioides, as demonstrated by the ability of these populations to respond to experimental vaccination and skin testing (spherulin, coccidioidin) to a similar extent as other ethnicities. ${ }^{8}$ Class II HLA-DRB1*1301 alleles have been associated with an increased risk for severe disseminated Coccidioides infection regardless of ethnicity; however, these alleles are not overrepresented in these patient populations. ${ }^{8}$

In patients with primary pulmonary coccidioidomycosis with no evidence of dissemination, guidelines generally recommend offering treatment to groups at high risk of dissemination, such as pregnant women and patients with diabetes mellitus. Given the high incidence of disseminated and severe disease in Black and Filipino patients, some guidelines endorse treatment of all cases of coccidioidomycosis in this patient population. ${ }^{8}$ No current data are available to help determine whether this broad treatment approach reduces the development of disseminated infection in these populations. Frequent monitoring for disease progression and/or dissemination 
involving clinical and laboratory reevaluation every 3 months for 2 years is highly recommended. ${ }^{8}$

Treatment generally is based on location and severity of infection, with disseminated nonmeningeal infection being treated with oral azole therapy (ketoconazole, itraconazole, or fluconazole) ${ }^{11}$ If there is involvement of the central nervous system structures or rapidly worsening disease despite azole therapy, amphotericin B is recommended at 0.5 to $0.7 \mathrm{mg} / \mathrm{kg}$ daily. In patients with disseminated meningeal infection, oral fluconazole $(800-1000 \mathrm{mg} / \mathrm{d})$ or a combination of an azole with intrathecal amphotericin B (0.01-1.5 mg/dose, interval ranging from daily to 1 week) is recommended to improve response. ${ }^{11}$

The differential diagnosis of cutaneous disseminated coccidioidomycosis is broad and includes other systemic endemic mycoses (histoplasmosis, blastomycosis) and infections (mycobacteria, leishmania). Lupus vulgaris, a form of cutaneous tuberculosis, presents as a palpable tubercular lesion that may coalesce into erythematous plaques, which may mimic endemic mycoses, especially in patients with risk factors for both infectious etiologies such as our patient. ${ }^{12}$ Disseminated histoplasmosis may present as polymorphic plaques, pustules, nodules, and ulcerated skin lesions, whereas disseminated blastomycosis characteristically presents as a crusted verrucous lesion with raised borders and painful ulcers, both of which may mimic coccidioidomycosis. ${ }^{13}$ Biopsy would reveal the characteristic intracellular yeast in Histoplasma capsulatum and broad-based budding yeast form of Blastomyces dermatitidis in histoplasmosis and blastomycosis, respectively, in contrast to the spherules seen in our patient's biopsy. ${ }^{13}$ Localized cutaneous leishmaniasis initially develops as a nodular or papular lesion and can progress to open ulcerations with raised borders. Biopsy and histopathology would reveal round protozoal amastigotes. ${ }^{14}$ Other diagnoses that should be considered include mycetoma, nocardiosis, and sporotrichosis. ${ }^{15}$ As the cutaneous manifestations of Coccidioides infections are varied, a broad differential diagnosis should be maintained, and probable environmental and infectious exposures should be considered prior to ordering diagnostic studies.

\section{REFERENCES}

1. Garcia Garcia SC, Salas Alanis JC, Flores MG, et al. Coccidioidomycosis and the skin: a comprehensive review. An Bras Dermatol. 2015; 90:610-619.

2. DiCaudo DJ. Coccidioidomycosis: a review and update. J Am Acad Dermatol. 2006;55:929-942; quiz 943-925.

3. DiCaudo DJ, Yiannias JA, Laman SD, et al. The exanthem of acute pulmonary coccidioidomycosis: clinical and histopathologic features of 3 cases and review of the literature. Arch Dermatol. 2006;142:744-746.

4. Blair JE. State-of-the-art treatment of coccidioidomycosis: skin and soft-tissue infections. Ann NY Acad Sci. 2007;1111:411-421.

5. Crum NF, Lederman ER, Stafford CM, et al. Coccidioidomycosis: a descriptive survey of a reemerging disease. clinical characteristics and current controversies. Medicine (Baltimore). 2004;83:149-175.

6. Borchers AT, Gershwin ME. The immune response in coccidioidomycosis. Autoimmun Rev. 2010;10:94-102.

7. Smith CE, Beard RR. Varieties of coccidioidal infection in relation to the epidemiology and control of the diseases. Am J Public Health Nations Health. 1946;36:1394-1402.

8. Ruddy BE, Mayer AP, Ko MG, et al. Coccidioidomycosis in African Americans. Mayo Clin Proc. 2011;86:63-69.

9. Louie L, $\mathrm{Ng} \mathrm{S}$, Hajjeh R, et al. Influence of host genetics on the severity of coccidioidomycosis. Emerg Infect Dis. 1999;5:672-680.

10. McCotter OZ, Benedict K, Engelthaler DM, et al. Update on the epidemiology of coccidioidomycosis in the United States. Med Mycol. 2019;57(suppl 1):S30-S40.

11. Galgiani JN, Ampel NM, Catanzaro A, et al. Practice guideline for the treatment of coccidioidomycosis. Infectious Diseases Society of America. Clin Infect Dis. 2000;30:658-661.

12. Khadka P, Koirala S, Thapaliya J. Cutaneous tuberculosis: clinicopathologic arrays and diagnostic challenges. Dermatol Res Pract. 2018;2018:7201973.

13. Smith JA, Riddell JT, Kauffman CA. Cutaneous manifestations of endemic mycoses. Curr Infect Dis Rep. 2013;15:440-449.

14. Scorza BM, Carvalho EM, Wilson ME. Cutaneous manifestations of human and murine leishmaniasis. Int J Mol Sci. 2017;18:1296. 